\title{
THE ROLE OF MICROBIAL FILMS IN FERMENTATION
}

\author{
B. AtKINSON
}

Department of Chemical Engineering, University College of Swansea, UK.

\begin{abstract}
Microbial mass can be said to occur in one of two states; as 'flocs' freely suspended in an aqueous medium, or as a 'film' adhering to a support surface. Industrial fermenter configurations, at least since the development of deep-tank fermentation, have almost invariably been based on the use of microbial flocs, and the occurrence of a visible microbial film during fermentation is generally avoided. Exceptions include the 'trickling filter' in the waste water treatment industry, "vinegar' towers, animal tissue culture and microbial leaching.

Under the stimulus of increasing industrialization, aerobic fermenters evolved from configurations based upon surface growth at the gas-liquid interface to the use of suspended cultures in stirred-tank arrangements. The application of this latter configuration to continuous operation is subject to a number of basic disadvantages, particularly 'wash-out' and the difficulties inherent in the provision of an appropriate environmental history for the microbial mass. These disadvantages can to some extent be overcome with fermenter configurations based upon the use both of microbial films and flocs.

The kinetics of microbial films are now sufficiently well understood, at least for growth-associated systems, to justify inclusion in the conventional theories for both batch and continuous fermentation. Such theoretical treatments illustrate some of the advantages of using microbial films in terms of increased productivity, absence of wash-out, etc., as well as suggesting the magnitude of the surface area: fermenter volume ratios necessary in particular cases.
\end{abstract}

\section{NOMENCLATURE}

$A$ dimensionless parameter (defined by equation 4 )

$A_{\text {s }} \quad$ area of support surface per unit liquid volume

$A_{\mathrm{w}} \quad$ area of biologically active area per unit liquid volume

$B$ dimensionless parameter (defined by equation 4)

C limiting substrate concentration

$C_{\mathrm{I}} \quad$ limiting substrate concentration fed to a fermenter

$C^{*}$ limiting substrate concentration at interface between microbial mass and adjacent solution

$C_{1}^{\prime}$ limiting substrate concentration entering second

$\mathbf{L}^{-1}$

$\mathrm{L}^{-1}$ stage of a tubular fermenter

$F \quad$ volumetric flow-rate to a fermenter

$G \quad$ specific growth rate
$\mathrm{ML}^{-3}$

$\mathrm{ML}^{-3}$

$\mathrm{ML}^{-3}$

$\mathrm{ML}^{-3}$

$\mathrm{L}^{3} \mathrm{~T}^{-1}$

$\mathrm{T}^{-1}$ 


\section{B. ATKINSON}

\begin{tabular}{|c|c|c|}
\hline$h$ & mass transfer coefficient & $\mathrm{LT}^{-1}$ \\
\hline$k_{1}$ & biological rate (equation) coefficient & $\mathrm{T}^{-1}$ \\
\hline$k_{2}$ & biological rate (equation) coefficient & $L^{-1}$ \\
\hline$k_{3}$ & biological rate (equation) coefficient & $\mathbf{M}^{-1} \mathbf{L}^{3}$ \\
\hline$K_{\mathrm{s}}$ & Monod coefficient & $\mathrm{ML}^{-3}$ \\
\hline$K_{(1)}$ & first order overall rate coefficient & $\mathrm{LT}^{-1}$ \\
\hline$L$ & 'wet' microbial film thickness & $\mathbf{L}$ \\
\hline$M$ & concentration of 'dry' microbial mass & $\mathrm{ML}^{-3}$ \\
\hline$M_{1}$ & $\begin{array}{l}\text { initial concentration of 'dry' microbial mass fed to a } \\
\text { fermenter }\end{array}$ & $\mathrm{ML}^{-3}$ \\
\hline$M_{1}^{\prime}$ & $\begin{array}{l}\text { microbial mass entering second stage of a tubular } \\
\text { fermenter }\end{array}$ & $\mathrm{ML}^{-3}$ \\
\hline$N$ & $\begin{array}{l}\text { flux of substrate at interface between microbial } \\
\text { mass and adjacent solution }\end{array}$ & $\mathrm{ML}^{-2} \mathrm{~T}^{-1}$ \\
\hline $\operatorname{Pr}$ & productivity & $\mathrm{ML}^{-3} \mathrm{~T}^{-1}$ \\
\hline $\operatorname{Pr}_{(\mathrm{d})}$ & dimensionless productivity & - \\
\hline$Q$ & volumetric flow-rate per unit cross-sectional area & $\mathrm{LT}^{-1}$ \\
\hline$Q^{\prime}$ & $\begin{array}{l}\text { volumetric flow-rate per unit cross-sectional area in } \\
\text { the second stage of a tubular fermenter }\end{array}$ & $\mathrm{LT}^{-1}$ \\
\hline$R$ & specific rate of removal of substrate & $\mathrm{T}^{-1}$ \\
\hline$\left(S_{\mathrm{i}} K_{\mathrm{i}}\right)$ & yield coefficient & - \\
\hline$V$ & liquid volume in a fermenter & $\mathbf{L}^{3}$ \\
\hline$W$ & recycle parameter (defined by equation 16) & - \\
\hline$z$ & distance co-ordinate in the direction of flow & $\mathrm{L}$ \\
\hline$Z$ & fermenter length & $\mathrm{L}$ \\
\hline$\alpha$ & $\begin{array}{l}\text { dimensionless flow-rate parameter (defined by } \\
\text { equation 5) }\end{array}$ & - \\
\hline$\beta$ & dimensionless area parameter (defined by equation 6) & - \\
\hline$\gamma$ & microbial concentration coefficient & - \\
\hline$\varepsilon$ & parameter (defined by equation 29) & $\mathrm{ML}^{-3} \mathrm{~T}^{-1}$ \\
\hline$\kappa$ & endogenous respiration coefficient & $\mathrm{T}^{-1}$ \\
\hline$\lambda$ & effectiveness factor & -- \\
\hline$\rho_{0}$ & density of cells ( $d r y$ weight per unit wet volume) & $\mathrm{ML}^{-3}$ \\
\hline$\phi_{\mathrm{P}}$ & $\begin{array}{l}\text { re-cycle ratio } \\
\text { dimensionless }\end{array}$ & - \\
\hline
\end{tabular}

\section{INTRODUCTION}

A reasonable working hypothesis for the interaction between microorganisms and surfaces, suggests that any surface in contact with a nutrient medium which contains suspended microorganisms will, in time, become biologically active due to their adhesion. In due course they will develop into a continuous microbial layer. While there is a wealth of evidence both direct and circumstantial in support of this hypothesis ${ }^{1-3}$, none seems to exist to the contrary. The mechanism of microbial adhesion, the important characteristics both of the surfaces and of the microorganisms, merits further study. On a micro-scale chemical modification of the surface by the microorganisms cannot be ruled out. 
The converse of the above hypothesis, i.e. the complete removal of a microbial layer (sterilization of the surface) by the normal fluid mechanical forces in a fermenter, is unlikely.

It follows that microbial films are a feature of every fermenter. The performance of a number of fermenters, e.g., the 'trickling' filter ${ }^{4}$, the bio-disc ${ }^{5}$, and the 'quick' vinegar process ${ }^{6}$, is dominated by the kinetics of microbial mass in the form of a film. This is also true of a number of processes operated on a somewhat smaller scale, e.g., animal tissue culture ${ }^{7}$, and of some processes at present in the development stage, e.g., microbial leaching ${ }^{8}$.

In the laboratory microbial-film fermenters provide a very useful tool for the study of microbial kinetics since the microbial-film thickness can be controlled with relative ease ${ }^{9}$. In contrast, when using a continuous stirredtank fermenter (CSTF) in the laboratory the presence of microbial films is often a considerable nuisance because they are difficult to control and present problems with the interpretation of experimental results. Since the microbial mass in a fermenter exists both in the form of flocs and films, both contribute to the performance of the fermenter. However, the respective contributions depend largely upon the total volumes of both the flocs and the films. In the case of microbial films the hold-up depends upon the area per unit volume $\left(A_{\mathrm{s}}\right)$ and the thickness of the film $(L)$. In a particular fermenter, whether batch or continuous, either form of microbial hold-up may be dominant or both forms may contribute significantly to the performance of the fermenter.

For a given substrate-microbe system the kinetics are fixed. It follows that the next most significant factor which influences the extent of the hold-up of microbial flocs is the flow-rate. In contrast, the hold-up of microbial film is unaffected by the flow rate but depends upon the surface area provided and the equilibrium microbial-film thickness. With stirred tank fermenters the surface to volume ratios are small for reasons associated with complete mixing ${ }^{10}$. Thus the contribution of the microbial flocs can be expected to dominate, particularly in batch fermentation. However, at high flow-rates it can be anticipated that the microbial film will dominate. Thus it can be stated, providing the basic hypothesis is correct, that due to the presence of microbial films the performance of a CSTF will be finite at all flowrates.

Since microbial films can play an important part in the performance of a fermenter due either to the extent of the surface area provided or to the flowrate adopted, the equilibrium microbial-film thickness and the method of control of films becomes of considerable importance. Furthermore, not only is it necessary to have knowledge of the kinetics of microbial flocs for the design of fermenters, but knowledge of the kinetics of microbial films can also be necessary.

The antithesis of the above situation lies in the interpretation of experimental results obtained in the laboratory. Before a theory can be applied, it has to be ascertained which form of microbial hold-up is dominant. Or, since fermenter configurations based upon the dominance of microbial flocs are usually used, it is necessary to be quite certain that the microbial films present are not making a significant contribution to the measured performance. 


\section{B. ATKINSON}

\section{THE CONTROL OF MICROBIAL FILMS}

In principle a microbial layer exposed to a sufficiently high substrate concentration will continue to grow indefinitely. If the rate of increase in the microbial-film thickness is small, then at any instant of time the concentration profile within the film will be sensibly the same as that in a microbial layer of the same, but constant, thickness. In these circumstances the variation of the microbial-film thickness and the performance of a fermenter can be completely described in terms of the kinetics of microbial films ${ }^{11}$.

Kornegay and Andrews ${ }^{12}$ have provided experimental data for the consumption of substrate by a 'growing' microbial film. From these data it may be deduced that the film thickness increases indefinitely while the rate of substrate consumption attains a constant value. At this stage the film may be described as effectively 'semi-infinite' in thickness. The concentration profiles within such films have been obtained experimentally by Bungay, Whalen and Saunders ${ }^{13}$. In practice as the film continues to grow, the gaseous respiration products deep in the film reach a level of such proportions that they come out of solution and form pockets of gas (Figure 1). The adhesion

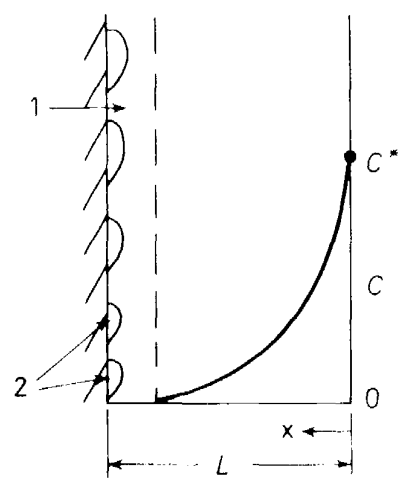

Figure 1. Conditions within a 'thick' microbial film. 1 Region of death, autolysis and gaseous products. 2 gas bubbles.

between the bulk of the microbial film and the supporting surface is impaired and ultimately the film falls away under the action of its own weight, leaving behind a thin layer of low viability. This layer then starts to 'grow' and it has been found that the films which subsequently develop often fail to regain their previous thickness. It may be that this phenomenon indicates a weakening of the adhesive forces because of the presence of dead organisms.

From the above comments it appears that microbial films are to a degree self-regulating. Reliance on this mechanism alone to achieve a constant hold-up of microbial film within a fermenter suffers from a number of major disadvantages:

(i) products formed in the depths of the film may be toxic or inhibitory to the viable organisms in the upper regions and may contaminate the liquid medium. 
(ii) microbial film which has become detached may cause blockages elsewhere in the fermenter.

(iii) the variations in the local microbial-film thickness can be large.

(iv) different areas of microbial mass break away at different times.

The first two of these disadvantages largely invalidate the use of the selfregulating mechanism of control for aseptic fermentation. The remainder present problems associated with design and operation of a fermenter since they lead to a dynamic variation about a mean performance. However, in the case of waste-water treatment, only (ii) is of real significance, since the product specification is described simply in terms of Chemical or Biological Oxygen Demand (COD or BOD) and the large size of the process units moderates the effects of (iii) and (iv). In such processes the effect of (ii) is diminished by the use of a relatively open support structure which allows the microbial mass to pass easily through the system. The worms and larvae which live on the microorganisms provide a second mechanism for the removal of microbial mass ${ }^{14}$.

Fermenter configurations in which the microbial flocs are dominant suffer from the operational limitations imposed by 'wash-out'. In contrast fermenters containing a fixed biological film avoid this problem and, providing the biological-film thickness can be controlled, a basis exists for fermenter configurations which are not limited to any particular range of flow-rates.

To date, fermenters based upon the use of a controlled biological-film thickness have found their use purely as a convenient laboratory configuration for the study of microbial kinetics ${ }^{9}$. In such investigations the biologicalfilm thickness is maintained constant by the purely mechanical procedure of hand-scraping, a technique which is hardly applicable to industrial operations. This procedure is restricted to fermenters in which the microbial film is readily accessible. For fermenter configurations based upon the packed column arrangement, the microbial-film thickness can be controlled by pcriodic washing with high-pressure water jets. At present this method has only been applied to a laboratory model of a 'trickling' filter ${ }^{15}$.

For aseptic operation it is desirable to have an essentially uniform and constant biological-film thickness on all the 'active' surfaces in the fermenter. In principle it may be possible to devise microbiological and biochemical methods for achieving such a constant biological-film thickness though for large scale operations it is reasonable to assume that this objective has to be achieved with the aid of hydrodynamic shear, mechanical devices, or both.

Figure 2 shows the build-up of microbial mass on an impeller taken from a 20 litre fermenter after 3 months of continuous operation with a mixed culture with zoogloeal characteristics. In this experiment the impeller was rotating at 500 r.p.m. Other observations on transfer lines containing microbial suspensions suggest that hydrodynamic shear alone is usually insufficient to control a microbial film and indeed often leads to a more compact film than would otherwise develop.

It follows that hydrodynamic shear has to be supplemented by mechanical action. The former is a feature of all fermenters as the contents are necessarily in a state of flow, while the latter could be provided by automatic mechanical scrapers ${ }^{16.17}$. There are considerable advantages in terms of reliability, 


\section{B. ATKINSON}

flexibility and simplicity if the mechanical action were provided without recourse to direct mechanical linkages. This could be achieved by abrasion due to the physical contact of solid surfaces. Such contacts are easily achieved by the use of relatively small, discrete, biochemically-inert particles as support surfaces for microbial growth. If these particles were maintained in suspension, the inevitable and frequent particle-particle contacts would

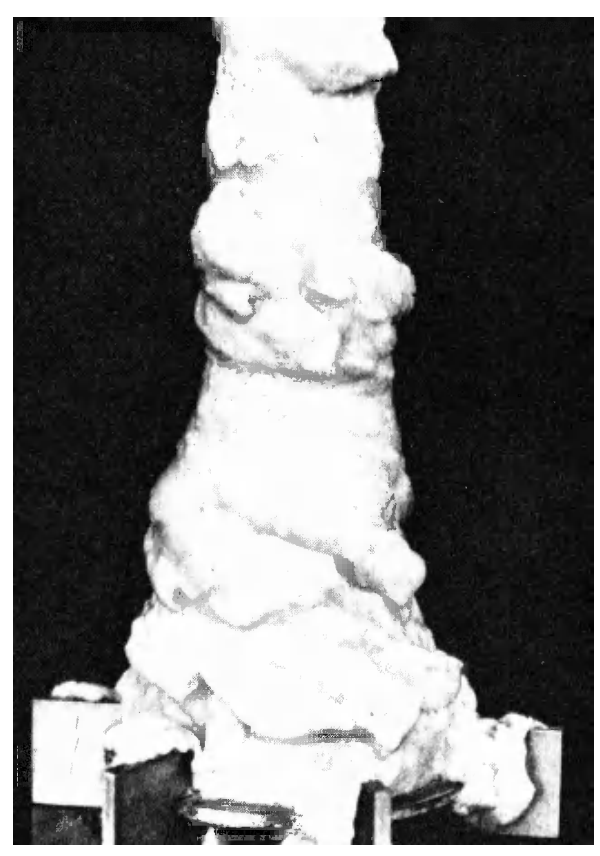

Figure 2. Growth on an impellor (rotation speed 500 r.p.m.).

cause the biological film to attain a dynamic steady-state between the growth of microbial mass and the forces of attrition.

Under the conditions described, the 'excess' microbial growth is transferred as flocs to the liquid phase and swept from the fermenter. These flocs would have a finite residence time within the fermenter and contribute to the overall performance.

Two arrangements are possible for fermenters of this type, a quantity of inert particles can be added to a conventional CSTF configuration (Figure 3) or alternatively the particles can be arranged in the form of a bed and fluidized (Figure 4), qualitatively at least, in the same manner as the purely biological particles in the Tower Fermenter ${ }^{18}$.

If the 'microbial-film fluidized-bed' fermenter were operated with a recycle stream (Figure 5), an improved performance would result. The physiological and biochemical characteristics of the microorganisms returned to the 


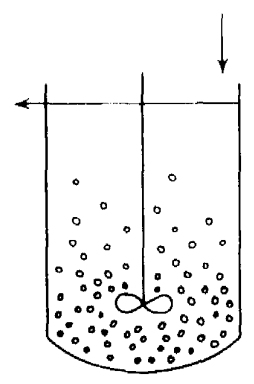

Figure 3. CSTF containing 'inert' particles.
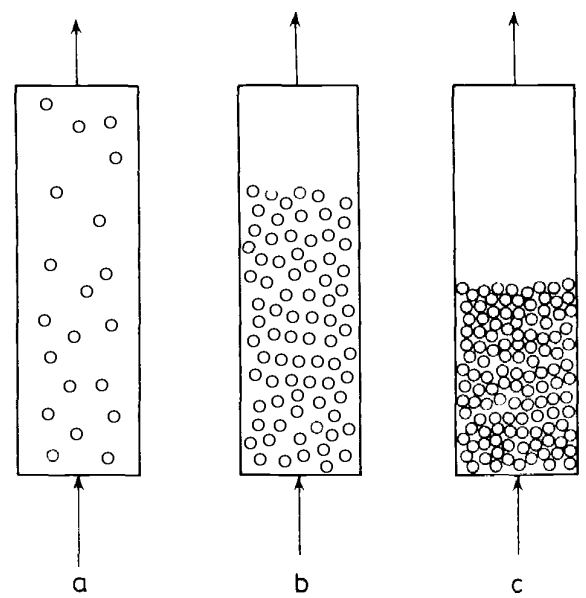

Figure 4. Tubular fermenter. a- elutriation; $\mathrm{b}-$ fluidized bed; $\mathrm{c}-$ fixed bed.

fermenter would naturally reflect the environmental conditions of the outlet rather than the inlet, and this may lead to additional difficulties. However the conditions in a tubular fermenter configuration, when operated at a high recirculation rate, approach those of a CSTF, i.e. the environmental conditions are sensibly constant throughout the liquid volume.

Thus microbial-film fermenters can be arranged either on stirred-tank or tubular fermenter principles and the selection of the appropriate configuration for a particular fermentation depends upon the same features of the fermentation which are relevant to the selection of the appropriate simple 'floc' fermenter configuration.

\section{THE KINETICS OF MICROBIAL FILMS}

The consumption of substrate by a microbial film is most conveniently expressed in terms of a flux evaluated at the interface between the film and 


\section{B. ATKINSON}

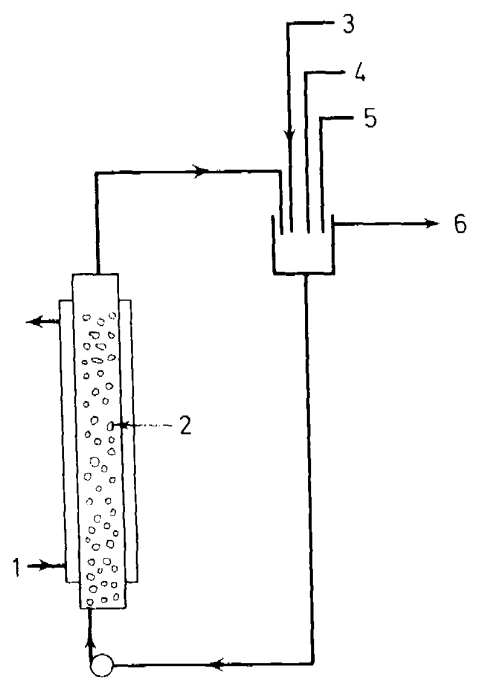

Figure 5. The completely mixed microbial film fermenter (CMMFF): 1 cooling water: 2 support particles: 3--feed $\left(\mathrm{F}, \mathrm{C}_{1}\right): 4-\mathrm{pH}$ control: 5--temperature control: 6 -products $\left(F, \mathrm{C}_{0} . M\right)$.

the substrate solution. This follows since at steady state all the substrate which passes through this interface is ultimately consumed by the microorganisms which make up the film. Thus the contribution of the microbial films to the total removal of substrate per unit fermenter volume is given by $N A_{\text {s }}$, where $N$ is the flux of substrate at the liquid-'solid' interface.

A knowledge of diffusion and biochemical reaction suggests that the magnitude of this flux depends upon the substrate concentration to which the film is exposed $\left(C^{*}\right)$, the thickness of the film $(L)$, and various kinetic parameters $\left(k_{1}, k_{2}\right.$ and $\left.k_{3}\right)$. This problem has been the focus of considerable attention in recent years. Atkinson and Daoud ${ }^{9.19}$ first provided a pseudoanalytical solution over a restricted range of the parameters and. more recently, Atkinson et $a l^{20.21}$ have extended the formulations to include all values of the parameters.

This 'biological rate equation' (Table 1) applies equally well to both films and flocs and contains as special cases most of the other equations which have been proposed ${ }^{21}$. The biological rate equation coefficients $k_{1}, k_{2}$ and $k_{3}$ are unique for a given substrate-microbe system, and of them, $k_{2}$, as may be deduced from Table 1 , reflects a diffusional limitation.

When a floc or film exhibits no diffusional limitation the effectiveness factor $\lambda$ is unity and $k_{2}$ is absent from the equations. In these circumstances the coefficients $k_{1}$ and $k_{3}$ can be related to the coefficients in the Monod equation for microbial kinetics,

i.e.

$$
K_{\mathrm{s}}=1 / k_{3}
$$


MICROBIAL FILMS IN FERMENTATION

Table 1 . The biological rate equation.

for films

$$
N=\lambda \frac{k_{1} L_{\mathrm{c}} C^{*}}{1+k_{3} C^{*}}=\lambda N_{\max } \frac{k_{3} C^{*}}{1+k_{3} C^{*}}
$$

for flocs

$$
R=\lambda \frac{\left(k_{1} / \rho_{0}\right) C^{*}}{1+k_{3} C^{*}}=\lambda R_{\max } \frac{k_{3} C^{*}}{1+k_{3} C^{*}}
$$

where

$$
\begin{array}{ll}
\lambda=1-\frac{\tanh \left(k_{2} L_{\mathrm{c}}\right)}{k_{2} L_{\mathrm{c}}-}\left[\frac{\phi_{\mathrm{P}}}{\tanh \phi_{\mathrm{P}}}-1\right], & \phi_{\mathrm{P}} \leqslant 1 \\
\lambda=\frac{1}{\phi_{\mathrm{P}}}-\frac{\tanh \left(k_{2} L_{\mathrm{c}}\right)}{k_{2} L_{\mathrm{c}}}\left[\frac{1}{\tanh \phi_{\mathrm{P}}}-1\right], & \phi_{\mathrm{P}} \geqslant 1
\end{array}
$$

and

$$
\phi_{\mathrm{P}}=\frac{k_{2} L_{\mathrm{c}}}{\sqrt{2(1} \frac{k_{3}}{\left.C^{*}\right)}}\left[k_{3} C^{*}-\ln \left(1+k_{3} C^{*}\right)\right]^{-\frac{1}{2}}
$$

and

$$
G_{\max }=\left(S_{0} K_{0}\right) k_{1} / k_{3} \rho_{0}=\left(S_{0} K_{0}\right) R_{\max }
$$

where $\left(S_{0} K_{0}\right)$ is a yield coefficient and $\rho_{0}$ is the microbial density in terms of dry weight per unit wet volume.

The characteristics of the biological rate equation for microbial films are illustrated in Figure 6 for a given set of rate coefficients. From this Figure it

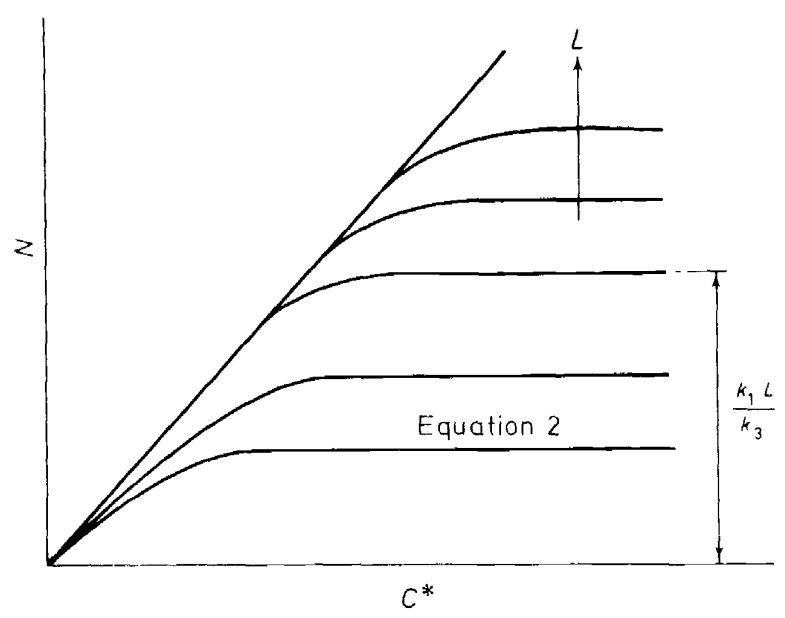

Figure 6. The biological rate equation for microbial films.

can be seen how, when the film is allowed to accumulate, the flux attains a limiting value when exposed to a given substrate concentration. 


\section{B. ATKINSON}

Experimental confirmation of the biological rate equation has been provided by Atkinson and coworkers ${ }^{11,19,22,23}$ using mixed microbial cultures with glucose and $\mathrm{NH}_{3}-\mathrm{N}$ as substrates in a biological film reactor. Data obtained by a number of other workers using pure cultures have also been shown to be equally well described by the equations ${ }^{23.24}$

\section{THE COMPLETELY-MIXED MICROBIAL FILM FERMENTER (CMMFF)}

\section{Effect of hiologically active area on conversion.}

The theory of Herbert ${ }^{25}$ for simple growth associated systems in a CSTF has been extended by Atkinson and Davies ${ }^{26}$ to include the effect of any microbial films which may be present in the fermenter. For the simple case when no 'solid' or liquid phase diffusional limitations are in evidence, the microbial kinetics given in Table 1 reduce to:

for flocs

$$
R=\frac{\left(k_{1} / \rho_{0}\right) C}{1+k_{3} C}=R_{\max } \frac{k_{3} C}{1+k_{3} C}
$$

for films

where $R_{\max }=k_{1} / k_{3} \rho_{0}$ and $N_{\max }=k_{1} L / k_{3}$

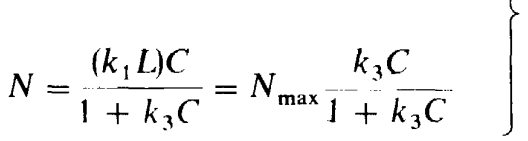

Inclusion of equation 2 in the laws of conservation applied to the CMMFF leads to the quadratic equation

$$
A\left(C / C_{1}\right)^{2}+B\left(C / C_{1}\right)-1=0
$$

where $0<C / C_{1} \leqslant 1$; $C$ represents the substrate concentration in the fermenter and $C_{1}$ is the inlet substrate concentration.

In equation 3 , the coefficients are given by

where

$$
\left.\begin{array}{l}
A=k_{3} C_{\mathrm{I}}(1-1 / \alpha) \\
B=1-k_{3} C_{1}+\frac{1}{\alpha}\left(k_{3} C_{\mathrm{I}}+\beta\right)
\end{array}\right\}
$$

and

$$
\alpha=\frac{F / V(1+\kappa V / F)}{G_{\max }}
$$

$$
\beta=\frac{k_{1} L A_{\mathrm{s}}}{G_{\max }}
$$

In equation $5 \kappa$ is an endogenous respiration coefficient.

The parameters $\alpha$ and $\beta$ can be seen to be a dimensionless flow-rate parameter and a dimensionless microbial-film parameter.

When $A_{\mathrm{s}}$ is identically zero (a simple CSTF) the solution to equation 3 is given by

$$
C / C_{\mathbf{1}}=\begin{gathered}
1 \text { or }-1 / A \\
288
\end{gathered}
$$




\section{MICROBIAL FILMS IN FERMENTATION}

The simple CSTF represents an asymptote of the CMMFF and equation 7 is a dimensionless form of the equations given by Herbert.

The general solution to equation 3 is given in Figure 7. In this Figure equation 7 can be seen to delineate the region of interest, i.e. values of the dimensionless concentration $\left(C / C_{1}\right)$ between zero and unity. Unfortunately since the algebraic formulation of the coefficient $B$ includes both $\alpha$ and $\beta$, it is not easy to gain more than a general impression from Figure 7 of the

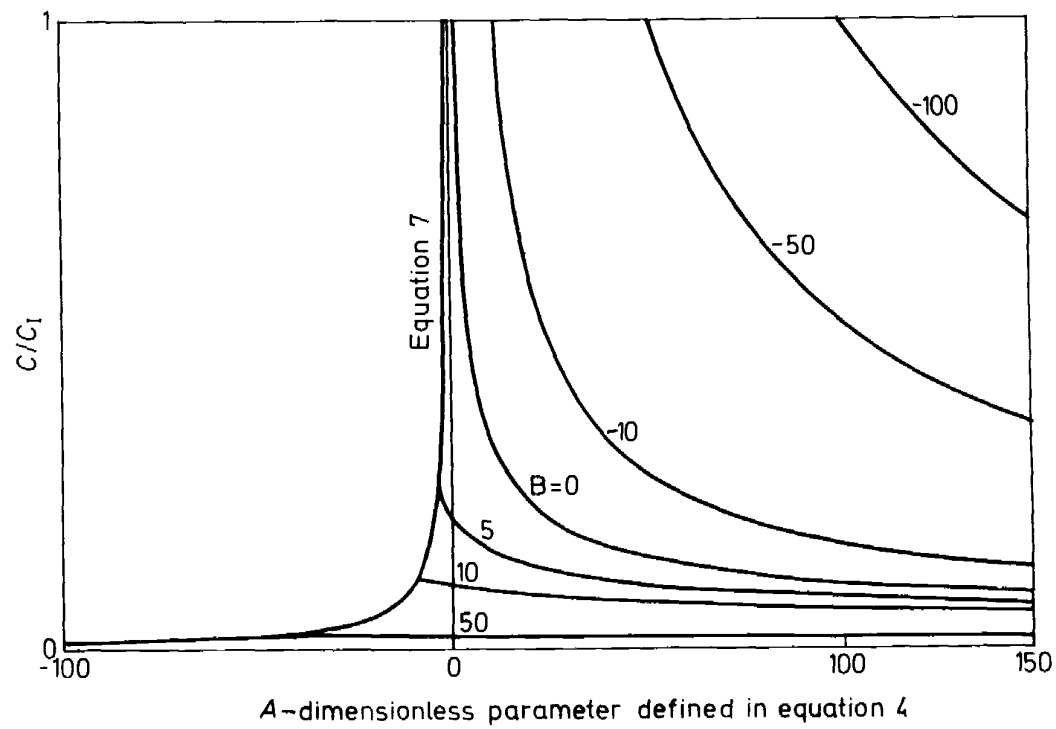

Figure 7. The performance characteristics of the CMMFF.

influence of changes in the flow-rate or the biologically-active area on the performance of a fermenter. Inspection of equations 3 and 4 indicates that the solution can be considered as

$$
C / C_{1}=g\left(\alpha, \beta, k_{3} C_{1}\right)
$$

This equation has been plotted in Figure 8 neglecting the term $\kappa V / F$, for a particular value of the parameter $k_{3} C_{\mathrm{I}}$. From this figure the absence of the wash-out phenomenon is evident for non-zero values of $\beta$, i.e. finite biologically-active areas.

\section{Influence of biologically active area on productivity}

The fermenter productivity, expressed as substrate consumed, is related to the substrate concentration by

$$
\operatorname{Pr}=F C_{\mathrm{I}}\left(1-\frac{C}{C_{\mathrm{I}}}\right) / V
$$




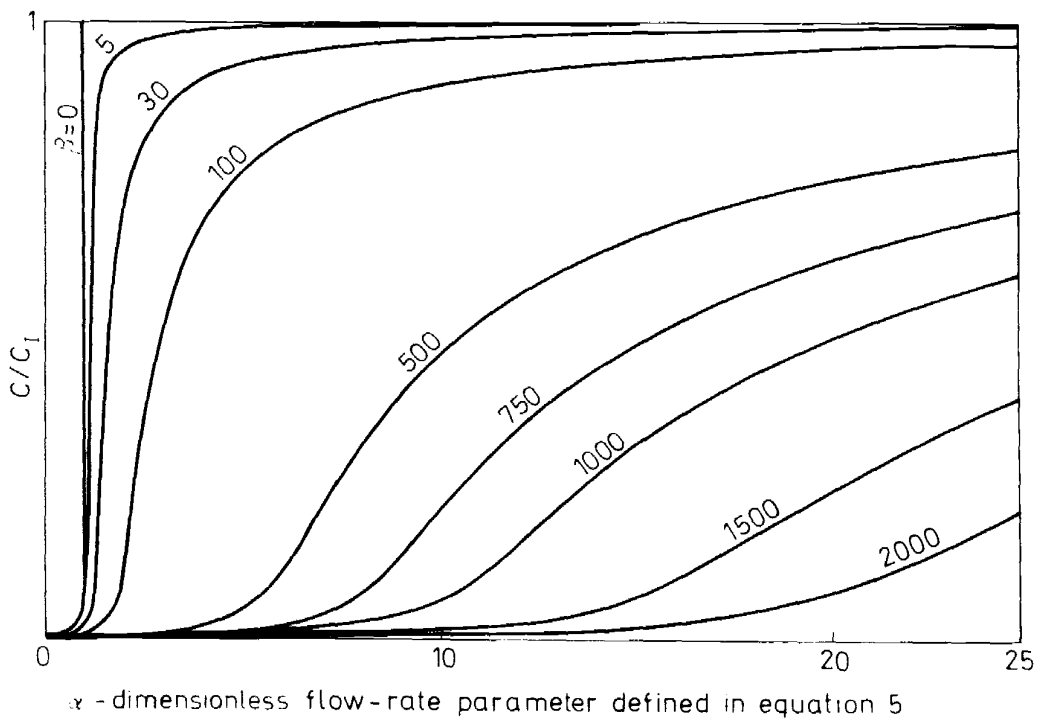

Figure 8 . The effect of biologically-active surface on the CMMFF $\left(k_{3} C_{1}=100\right)$

or

$$
P r_{(\mathrm{d})}=\alpha\left(k_{3} C_{1}\right)\left(1-\frac{C}{C_{1}}\right)
$$

where $\operatorname{Pr}_{(\mathrm{d})}$ is a dimensionless productivity given by

$$
\operatorname{Pr} \frac{k_{3}}{G_{\max }}(1+\kappa V / F)
$$

Equation 10 has been plotted in Figure 9. It can be seen that 'wash-out' is a feature only of a fermenter which has either sterile surfaces or is devoid of surface area. Thus in the mathematical sense 'wash-out' is unlikely to occur in real fermenters. However, when $A_{\mathrm{s}}$ is low, e.g. in conventional continuous stirred-tank fermenters, then a fair approximation to this condition is evident. From Figure 9 it can be seen that by increasing $A_{\mathrm{s}}$ the problems associated with the wash-out or near wash-out condition can be avoided.

The productivity curves shown in Figure 9 still pass through a maximum. However, this maximum is less sharp, it occurs at increased flow-rates and is of greater magnitude the larger the value of $A_{\mho}$, Indeed, it can be argued that to obtain near maximum productivity in a simple CSTF there is a flow-rate below which the fermenter should be operated to ensure stable conditions. In contrast for the CMMFF the shape of the productivity curve encourages operation beyond a minimum flow-rate for any given value of $A_{\mathrm{s}}$.

At large flow-rates the substrate concentration in the CMMFF approaches the inlet concentration (Figure 8). Under these conditions the residence time of the flocs is very small, but unlike the CSTF the CMMFF still exhibits a significant performance. Indeed for large areas (large values of $\beta$ ) the maxi- 
MICROBIAL FILMS IN FERMENTATION

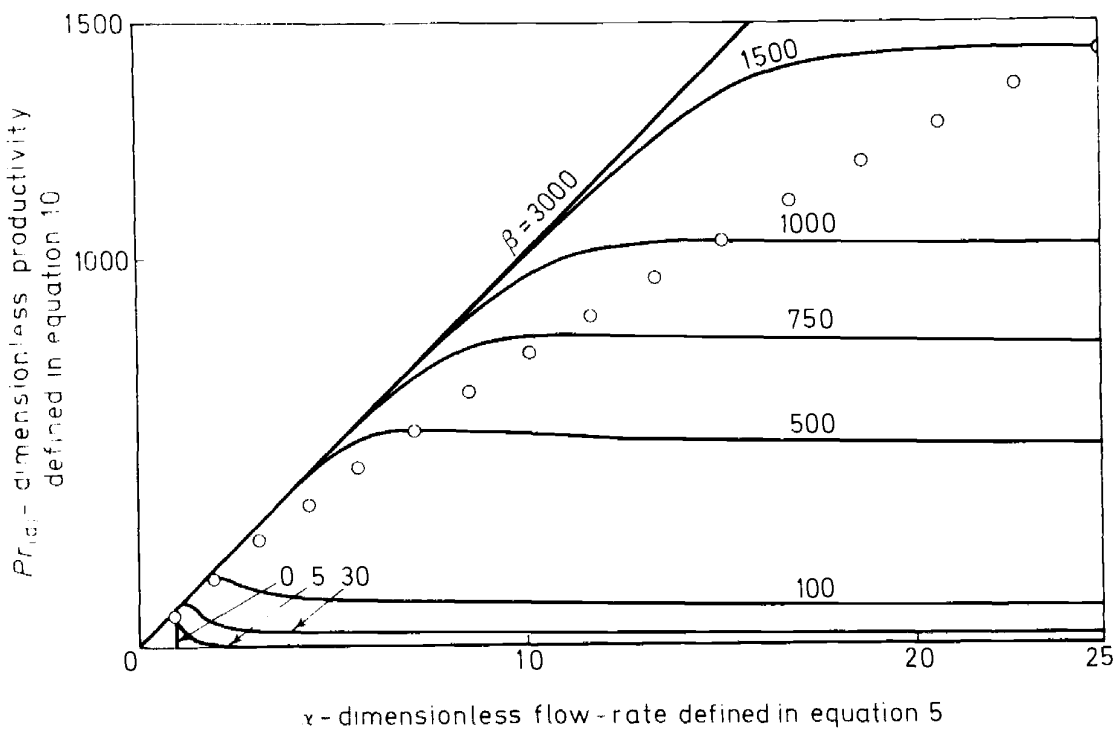

Figure 9. The productivity of the CMMFF. O locus of $\operatorname{Pr}_{(\mathrm{d}) \max }$

mum productivity and the productivity at large flow-rates are very similar (Figure 9).

As the flow-rate is increased

and

$$
\left.\begin{array}{l}
C \rightarrow C_{\mathrm{I}} \\
P r \rightarrow N A_{\mathrm{s}}
\end{array}\right\}
$$

or

$$
\sum_{F \rightarrow \text { large }} \operatorname{Pr} \rightarrow \frac{k_{1} L_{1} C_{1}}{1+k_{3} C_{1}} A_{\mathrm{s}}
$$

Equation 12 can be expressed in terms of the dimensionless productivity i.e.

$$
\operatorname{Pr}_{(\mathrm{d})} \rightarrow \beta \frac{k_{3} C_{1}}{1+k_{3} C_{1}}
$$

and

$$
\underbrace{}_{F, \overline{C_{1}} \rightarrow \text { large }} \operatorname{Pr}_{(\mathrm{d})} \rightarrow \beta
$$

The asymptotic nature of the productivity curves can be seen from Figure 9. 


\section{B. ATKINSON}

\section{Inclusion of a microbial concentration stage}

A recycle stream incorporating a microbial concentration stage can be accounted for as with a simple CSTF. Equations 3 and 4 still provide the design equations but the coefficients $\alpha$ and $\beta$ are given by

$$
\begin{gathered}
\alpha=\frac{F / V(W+\kappa V / F)}{G_{\max }} \\
\beta=W A_{\mathrm{s}} k_{1} L / G_{\max }
\end{gathered}
$$

where $W=1-\tau(\gamma-1) ; \gamma$ is the microbial concentration ratio $(>1)$ and $\tau$ the recycle ratio.

The data given in Figures 7-9 cover this situation. It can be deduced that a microbial concentration stage improves the fermenter performance and that there are no limitations on either $\gamma$ or $\tau$ equivalent to those associated with the simple $\mathrm{CSTF}^{25}$.

\section{THE CHARACTERISTICS OF A LABORATORY-SCALE CSTF}

The extent of the 'surface activity' depends upon the area involved, the thickness of the microbial layer and the substrate concentrations in the nutrient medium as well as the biological system parameters. The parameter $\beta$ defined in equation 6 characterizes this activity.

$$
\beta=\frac{\rho_{0} k_{3}}{\left(S_{0} K_{0}\right)} L A_{\mathrm{s}}
$$

i.e., the product of a geometric factor, a biological factor and a physical parameter.

In view of the influence of $\beta$ on the performance characteristics of a continuous fermenter, it is appropriate to give some consideration to the values of this parameter which might be expected to occur within a laboratoryscale CSTF.

A typical value of $A_{\mathrm{s}}$ for a small fermenter is $0.5 \mathrm{~cm}^{-1}$. For a carbohydrate substrate the orders of magnitude of the biological parameters $\rho_{0}, k_{3}$ and $\left(S_{0} K_{0}\right)$ are $1 \mathrm{~g} \mathrm{~cm}^{-3}, 1 \mathrm{mg}^{-1} 1$ and 0.5 respectively ${ }^{27}$. Substituting these values into equation 6 gives a value of $\beta$ of the order of $10^{6}$ times the biological film thickness, i.e.

$$
\beta=\left(10^{6}\right) L
$$

where $L$ is the thickness of the microbial layer in centimetres.

To evaluate $\beta$ further, a range of typical values of $L$ is necessary. On the basis of the present hypothesis the minimum value of the biological film thickness is $1 \mu$; this corresponds to a microbial layer of single cell proportions.

Atkinson et al. ${ }^{22}$ have carried out a series of studies using a biologicalfilm reactor containing microbial films of essentially constant thickness. This was achieved by vigorous mechanical scraping of the active surface. The result was a biologically-active surface with a bacterial layer scarcely visible to the eye. The mixed microbial culture had zoogloeal characteristics 
and consisted predominantly of gram-positive rod-shaped bacteria, but with significant numbers of gram-negative rod-shaped bacteria. A small number of gram-positive cocci and pseudo-mycelial yeast were also present. The microbial-film thickness was calculated from kinetic data as $7.04 \times 10^{-3} \mathrm{~cm}$.

These values of the biological-film thickness, i.e. 1 and 70 microns, indicate values of $\beta$ in the range $100-1000$.

For a value of $k_{3} C_{1}$ of 100 , Figure 8 indicates that the lower end of this range deviates significantly from the simple CSTF theory, i.e. $\beta=0$, and that the higher values result in quite different levels of performance.

In practical terms these observations suggest that a determination of $L$ is a necessity for all studies involving a CSTF, at least when using carbohydrate substrates. The difficulty inherent in this requirement lies in the small magnitudes involved, as well as the fact that the biological-film thickness undoubtedly varies over the solid surface due to a varying degree of hydrodynamic shear.

In the case of microbe systems which lead to relatively thin microbial films, and therefore small values of $\beta$, it is possible to avoid the necessity for a determination of $L$, by the use of a sufficiently large value of $k_{3} C_{\mathrm{r}}$. The influence of $C_{\mathrm{I}}$ is shown in Figure 10 when $\beta$ is 100 . The larger the value of

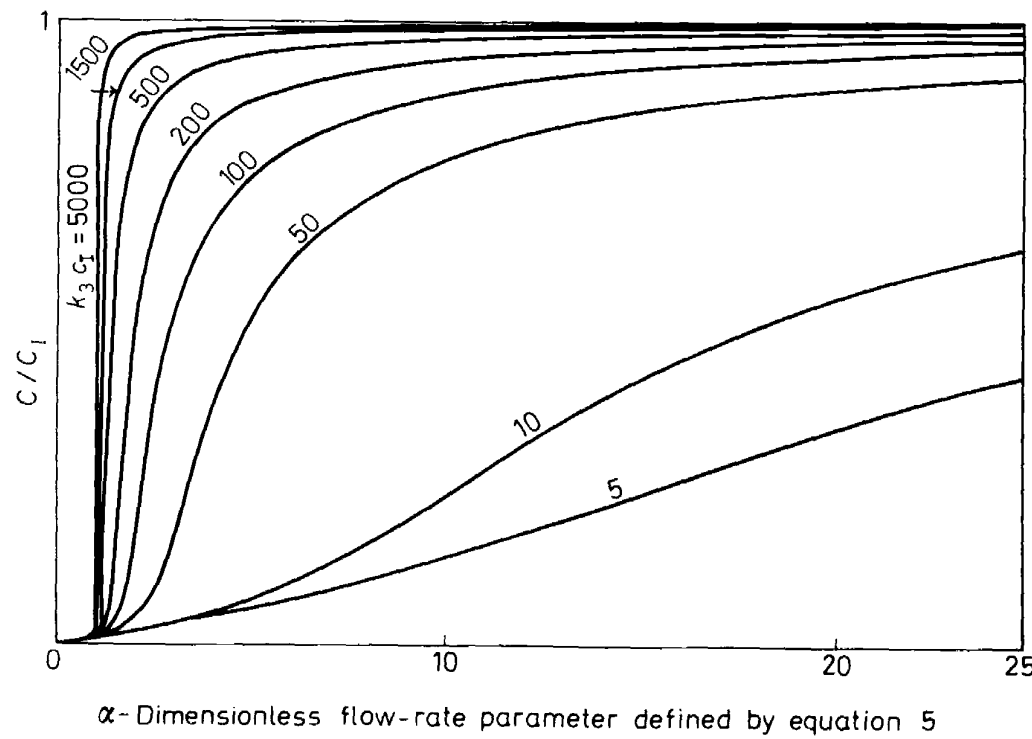

Figure 10. The effect of inlet concentration on the CMMFF $(\beta=100)$.

$C_{1}$ the closer the approximation to the simple CSTF theory. The nature of the curves is such that at sufficiently high inlet concentrations an approximation to the theoretical wash-out flow-rate could be obtained by imposition of the simple CSTF theory, i.e. ignoring the contribution of the microbial film to the fermenter performance. 


\section{B. ATKINSON}

\section{The CSTF with particles added}

As illustrated in Figure 3 particles can be added to a CSTF and this leads to a very convenient procedure for changing the area $A_{\mathrm{s}}$ while maintaining the fermentation.

In Figure 11 are given data obtained using a 2 litre fermenter and the substrate-microbe system of Atkinson et al. ${ }^{22}$. This system consisted of a mixed microbial culture and a synthetic feed containing glucose, potassium

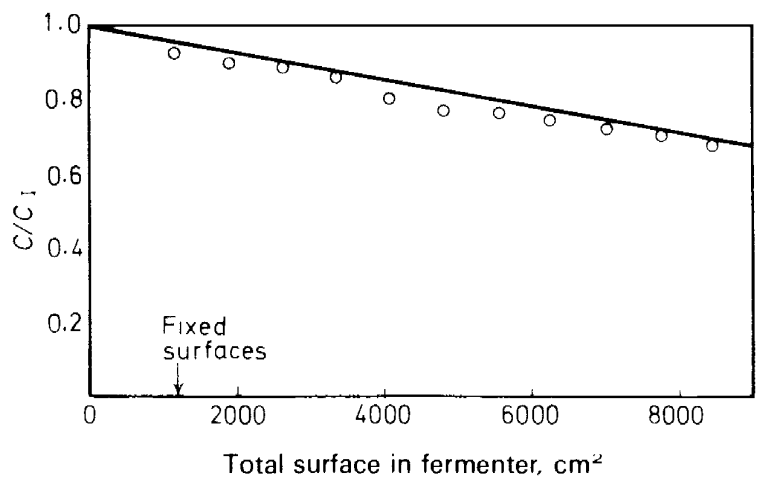

Figure 11. CSTF with particles added effect of arca. $\mathrm{O}-$ experimental results : $-L=0.25 \mathrm{~mm}$.

nitrate and ammonium hydrogen phosphate in such proportions that glucose was the rate controlling reactant. Anaerobic conditions and nonaseptic operation were used throughout the experiments.

These experiments were carried out with glass ballotini, grade No. 10 (mean diameter $0.28 \mathrm{~mm}$ ), and the experimental methodology allowed a steady state to be established before the addition of further particles. The additions were made in increments of $10 \mathrm{~g}$ i.e. areas of $732 \mathrm{~cm}^{2}$. The total immersed surface area of the fermenter and its ancillary equipment was $1168 \mathrm{~cm}^{2}$. The flow-rate used throughout was $37 \mathrm{ml} \mathrm{min}^{-1}$ and the fermenter was operated at $\mathrm{pH} 7$ and $22 \mathrm{C}$ with an inlet concentration of $1100 \mathrm{mg}^{-1}$. The glucose concentration was determined by the glucose oxidase test method.

The results given in Figure 11 indicate an essentially linear relationship between the substrate uptake and the surface area. When the conversion efficiency of the fermenter in the absence of particles is recorded as a data point, extrapolation of the data to zero surface area indicates that a conversion efficiency of zero would be expected. This suggests that the flow-rate used is in excess of the theoretical wash-out flow.

The biological system parameters for the substrate microbe system under eonsideration have been provided by Atkinson $e a^{a l}{ }^{11,22}$ as:

$$
\left.\begin{array}{rl}
\left(S_{0} K_{0}\right) & =0.731 \\
k_{1} & =0.1624 \mathrm{~s}^{-1} \\
k_{3} & =1.706 \times 10^{5} \mathrm{~g}^{-1} \mathrm{~cm}^{3}
\end{array}\right\}
$$


The theoretical wash-out flow is given by equations 4 and 7 as

$$
F_{\mathrm{w} / 0}=\left(S_{0} K_{0}\right) \frac{k_{1}}{k_{3} \rho_{0}} V=G_{\max } V
$$

Insertion of the data given in equation 17 into equation 18 indicates that the flow-ratc used in the experiments was two orders of magnitude greater than the theoretical wash-out flow. In these circumstances it can be anticipated that the asymptote indicated by equation 11 provides an appropriate basis for discussion, i.c.

or

$$
\left.\begin{array}{c}
F\left(C_{1}-C\right)=\frac{k_{1} L}{k_{3}} A_{\mathrm{s}} \\
1-\frac{C}{C_{1}}=\frac{k_{1} L}{k_{3}} \frac{A_{\mathrm{s}}}{F C_{1}}
\end{array}\right\}
$$

Equations 19 suggest a linear relationship between $C / C_{1}$ and $A_{\mathrm{s}}$ with a slope of $k_{1} L / k_{3} F C_{1}$. The theoretical line plotted in Figure 11 corresponds to the data given in equation 17 and a microbial-film thickness of $0.25 \mathrm{~mm}$. This thickness while not confirmed by direct experiment is, at the very least, of the correct magnitude.

\section{THE OPERATING CHARACTERISTICS OF A CMMFF}

\section{Apparatus}

The apparatus used as a CMMFF is shown diagrammatically in Figure 5. The main features include a vertical tubular section containing $0.61 \mathrm{~cm}$ diameter glass beads and a reservoir containing nutrient inlets, overflow weir and control devices. Circulation of the fermenter contents was achieved by means of a centrifugal pump.

The fluidized-bed section comprised a $100 \mathrm{~cm}$ length of $4.5 \mathrm{~cm}$ diameter glass tube and contained approximately 8000 etched glass beads.

\section{Experimentation}

The microbiological and chemical systems were those employed previously with the CSTF. Anacrobic conditions and non-aseptic operation were used again, and the temperature and $\mathrm{pH}$ levels were maintained at $27^{\circ} \mathrm{C}$ and 7 respectively. The inlet concentration used was $1000 \mathrm{mg} \mathrm{l}^{-1}$. The degree of fluidization was maintained constant throughout the experimentation by operating at all times with a bed expansion of $8 \mathrm{~cm}$.

\section{Observations}

At low flow-rates visible microbial growth was confined largely to the liquid phase, which had the appearance of a homogeneous milky suspension. As the throughput $(F / V)$ was increased, a rapid development of microbial film was observed on the fixed surfaces of the fermenter. This growth occurred throughout the fermenter except in the region of the fluidized bed. If the 


\section{B. ATKINSON}

extent of bed expansion was reduced, i.e. a lower velocity of fluidization, visible growth quickly became apparent on the support particles.

The thicker microbial film attached to the fixed fermenter surfaces occasionally became detached and this resulted in large flocs (up to $5 \mathrm{~mm}$ ) in the circulating liquid. The periodic transfer of large quantities of microbial film into the liquid suspension produced a large degree of variability in the performance of the fermenter.

\section{Experimental results}

The results obtained are recorded in Table 2.

Table 2. Experimental results for the CMMFF. $\left(A_{\mathrm{q}}=3.4 \mathrm{~cm}^{-1}\right)$

Total internal surface area $13040 \mathrm{~cm}^{2}$

Area of spheres

$9340 \mathrm{~cm}^{2}$

Area of fixed surfaces

$3700 \mathrm{~cm}^{2}$

Liquid volume

3.84 litres

\begin{tabular}{crccc}
\hline $\begin{array}{r}\text { Flow-rate } \\
\left(\mathrm{ml} \mathrm{min}^{-1}\right)\end{array}$ & $\begin{array}{c}\text { glucose } \\
\text { concn. } \\
\left(\mathrm{mg} \mathrm{l}^{-1}\right)\end{array}$ & $\begin{array}{c}F / V \\
\left(\mathrm{~h}^{-1}\right)\end{array}$ & $\begin{array}{c}f \\
(-)\end{array}$ & $\begin{array}{c}\alpha \\
(-)\end{array}$ \\
\hline 3.8 & 0 & 0.0594 & 0 & 23.8 \\
7.2 & 0 & 0.112 & 0 & 44.9 \\
8.0 & 0 & 0.125 & 0 & 49.6 \\
13.7 & 2 & 0.214 & 0.002 & 85.0 \\
20 & 138 & 0.312 & 0.138 & 123.8 \\
35 & 210 & 0.547 & 0.210 & 216.9 \\
80 & 530 & 1.25 & 0.530 & 495.7 \\
160 & 680 & 2.50 & 0.680 & 992.8 \\
\hline
\end{tabular}

The flow-rate parameter $\alpha$ of equation 5 can be calculated using the data given in equation 17 providing the biological-film thickness is assumed to be of 'thin' proportions and endogenous respiration can be neglected. The assumption of a thin biological film is somewhat less appropriate outside the fluidized bed region of the fermenter but will suffice for present purposes.

The data given in Table 2 are plotted in Figure 12. Superimposed on this Figure are numerical data for

$$
f=g\left(\alpha, \beta, k_{3} C_{1}=170\right)
$$

From Figure 12 it can be seen that the data for the CMMFF are qualitatively, at least, related in the manner to be expected from the theory.

It is apparent from the data that the value of $\beta$ increases with $\alpha$. Since $\beta$ is proportional to the average biological-film thickness in the fermenter, this operating characteristic is compatible with the visual observations made during the course of the experiments and probably results from increased growth rates at the larger substrate concentrations.

From Figure 12 values of $\beta$ in the range 20000 to 60000 are indicated. These values correspond to biological-film thicknesses of $0.23-0.69 \mathrm{~mm}$. 


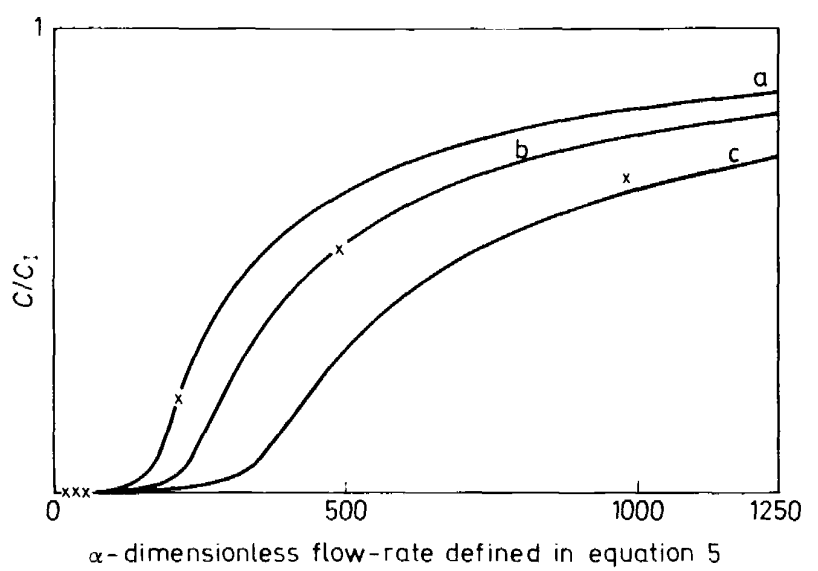

Figure 12. Experimental data for the CMMFF. $\times$-experimental results; theory $\left(k_{3} C_{1}=170\right)$ : curve $a-\beta=30000$, curve $b-\beta=40000$, and curve $c-\beta=60000$.

These thicknesses represent area-weighted averages between the fluidized particles and the fixed surfaces in the fermenter and. as such, compare favourably with the thickness estimated for the CSTF.

\section{THE TUBULAR MICROBIAL FILM FERMENTER}

\section{The fluidized-bed fermenter}

This fermenter is illustrated in Figure 4. Provided a combination of flow-rate and particle properties (size and density) is selected so that the particles experience just sufficient movement to control the biological-film thickness, an axial distribution of concentrations would result.

The thickness of the microbial layer within a given fermenter configuration can only be determined by experimentation. However, a knowledge of this. the system parameters $\left(k_{1}, k_{2}, k_{3},\left(S_{0} K_{0}\right),\left(S_{\mathrm{p}} K_{\mathrm{p}}\right)\right)$, the biologically active area per unit volume $\left(A_{\mathrm{s}}\right)$, and the liquid phase mass transfer coefficient, leads to a design procedure.

A mass balance on the limiting substrate over a differential length of fermenter for a simple plug-flow model, neglecting dispersion effects, gives:

$$
-Q \mathrm{~d} C=N A_{\mathrm{s}} \mathrm{d} z+R M \mathrm{~d} z
$$

where $Q$ is the volumetric flow-rate per unit cross-sectional area; $M$ is the local concentration of microbial flocs; $N$ and $R$ represent the consumption of substrate by the film and flocs respectively.

Providing there is no liquid phase resistance and the characteristic sizes of the films and flocs are small, $N$ and $R$ are given by equation 2 .

The local microbial and substrate concentrations can be related to the entrance conditions by an overall mass balance 


\section{B. ATKINSON}

i.e.

or

$$
\left.\begin{array}{c}
Q M=\left(S_{0} K_{0}\right) Q\left(C_{\mathrm{I}}-C\right) \\
M=\left(S_{0} K_{0}\right)\left(C_{\mathrm{I}}-C\right)
\end{array}\right\}
$$

Combination of equations 2, 21 and 22 leads to

$$
-Q \mathrm{~d} C=\left\{N A_{\mathrm{s}}+\left(S_{0} K_{0}\right) R\left(C_{\mathrm{I}}-C\right)\right\} \mathrm{d} z
$$

or

$$
\int_{C_{1}}^{C_{0}} \frac{\mathrm{d} C}{g(C)}=-\frac{Z}{Q}
$$

where

$$
\left.\begin{array}{l}
g(C)=\frac{\left(k_{1} L A_{\mathrm{s}}\right) C}{1+k_{3} C}+\frac{\left(S_{0} K_{0}\right)\left(C_{1}-C\right)\left(k_{1} / \rho_{0}\right) C}{1+k_{3} C} \\
\text { or } \quad \\
g(C)=\frac{k_{3} C\left(N_{\max } A_{4}+G_{\max } C_{1}\right)-G_{\max } k_{3} C^{2}}{1+k_{3} C}
\end{array}\right\}
$$

where

$$
N_{\max }=\frac{k_{1} L}{k_{3}} \text { and } G_{\max }=\left(S_{0} K_{0}\right) \frac{\left(k_{1} / \rho_{0}\right)}{k_{3}}
$$

The function $g(C)$ represents the local volumetric rate of substrate consumption $R_{\mathrm{v}}$ and is illustrated in Figure 13 with $A_{s}$ as a parameter. For a given biologically-active area in the fermenter the area under the curve between the limits $C_{1}$ and $C_{0}$ represents the integral of equation 24 . It can be seen that this

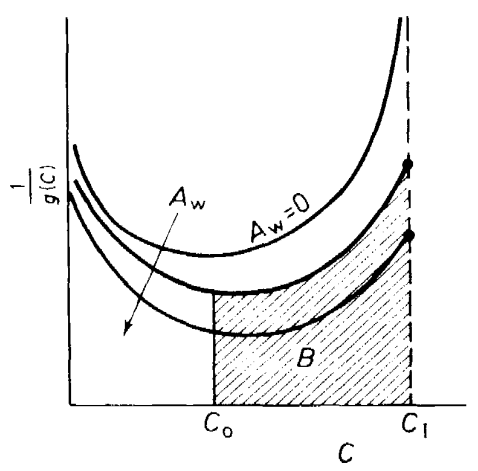

Figure 13. The effect of surface area in a tubular fermenter. $B$ area proportional to fermenter volume. 


\section{MICROBIAL FILMS IN FERMENTATION}

area, and therefore the fermenter volume, decreases if the amount of biologically-active surface is increased. In the absence of biologically-active surface the required fermenter volume is infinite since

$$
1 / g(C) \rightarrow \infty \text { as } C \rightarrow C_{\mathrm{I}} \text { for } A_{\mathrm{s}}=0
$$

This condition corresponds to wash-out for a tubular fermenter configuration containing only flocs and operated without re-circulation.

The solution to equation 24 is given by

$$
\frac{1}{\varepsilon k_{3}} \ln \frac{C_{0}}{C_{\mathrm{I}}}-\frac{G_{\max }+\varepsilon k_{3}}{\varepsilon k_{3} G_{\max }} \ln \frac{\varepsilon-G_{\max } C_{0}}{N_{\max } A_{\mathrm{s}}}=-\frac{Z}{Q}
$$

where

$$
\varepsilon=N_{\max } A_{\mathrm{s}}+G_{\max } C_{\mathrm{I}}
$$

\section{Film fermenter feeding a plug-flow fermenter}

To achieve a satisfactory continuous process it may be necessary to simulate the changes which take place in the equivalent batch fermentation. The reasoning behind this suggestion is associated with the apparent need to expose the organisms to a particular environmental history in order to produce a required biochemical product.

This requirement is not met completely by the microbial film fermenter described in the previous section, since the film growth in different parts of the fermenter has different histories. However the microbial mass, once it leaves the support surface, is exposed to exactly the same concentration variations as all the other flocs. For these reasons the microbial film fermenter may turn out to be applicable to all fermentations, but for the present it would appear sensible, at least for the more complex fermentations, to use the microbial film fermenter to generate microbial mass. This mass can then be fed to a tubular microbial floc fermenter. Such a configuration is illustrated in Figure 14; this consists of two tubular sections of different diameters mounted above one another, the lower section consisting of a controlled microbial film fermenter while the upper section is a simple tubular fermenter. The diameters of the two sections will usually be different, since they will have differing requirements as regards superficial flow-rate. In the one case fluidization of the support particles will dominate the design, while in the other the overall residence time of the nutrient medium will be all important.

For a simple growth associated system equation 28 provides the design equation for the section containing microbial films. Application of equations 21 and 22 to this section yields

$$
-Q^{\prime} \mathrm{d} C=R M \mathrm{~d} z
$$

and

$$
M=M_{\mathrm{I}}^{\prime}+\left(S_{0} K_{0}\right)\left(C_{\mathrm{I}}^{\prime}-C\right)
$$

where

$$
M_{\mathrm{I}}^{\prime}=\left(S_{0} K_{0}\right)\left(C_{\mathrm{I}}-C_{1}^{\prime}\right)
$$




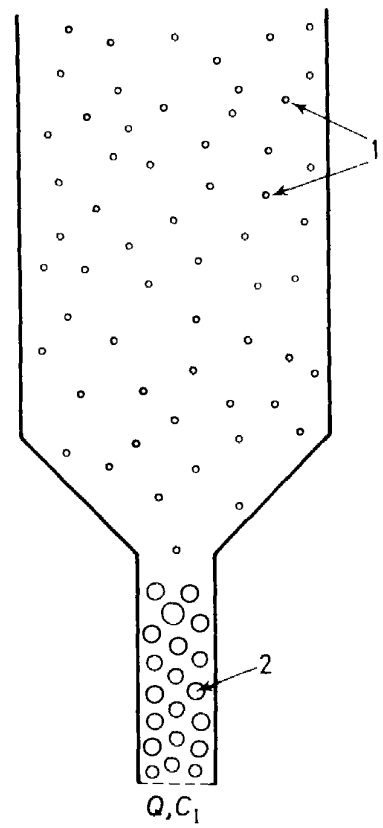

Figure 14. Fluidized-bed fermenter feeding a plug-flow fermenter. 1-flocs; 2-biologically active 'inert' particles.

In these equations $C_{1}^{\prime}$ and $M_{\mathrm{I}}^{\prime}$ represent the conditions on entry into the upper section and $Q^{\prime}$ is the volumetric flow-rate per unit cross-section of the upper section.

Combining equations 31 and 32

$$
M=\left(S_{0} K_{0}\right)\left(C_{\mathrm{I}}-C\right)
$$

and combining equations 30 and 33

where

$$
\int_{C_{1}}^{C_{0}} \frac{\mathrm{d} C}{g(C)}=-\frac{Z}{Q^{\prime}}
$$

$$
g(C)=\frac{G_{\max }\left(C_{1}-C\right) k_{3} C}{1+k_{3} C}
$$

The graphical procedure for the solution of equations 28 and 34 is illustrated in Figure 15.

\section{The trickle-flow fermenter}

-Uncontrolled' biological film thickness.

Fermenters containing an 'uncontrolled' biological film thickness are used primarily in the waste water treatment industry under the name 
MICROBIAL FILMS IN FERMENTATION

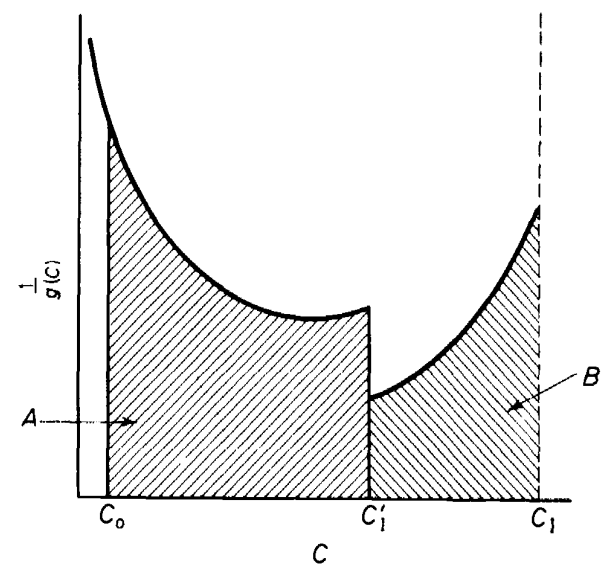

Figure 15. Design of an FBF feeding a PFF. $A$-- Area proportional to volume of upper section; $B$-area proportional to volume of lower section.

'trickling filter'4. The hold-up of microorganisms in the 'filter' is highly irregular and varies locally as a result of 'sloughing' (the break away of microbial film from the support surface) and the action of higher organisms. Liquid flows through the filter in the form of a film under the action of gravity. Usually the area wetted by the liquid film is rather less than the available biologically-active area. Growth takes place in the regions where nutrients are supplied and the increase in microbial-film thickness causes the liquid to be displaced laterally. The result is a complex interaction between the microbial kinetics, the microbial-film thickness and the wetted area. Fortunately a number of approximations are possible and these provide a basis for a kinetic description of the filters. Thus, (a) although the same biologically active area is not wetted all the time the extent of the wetted area is largely constant, and (b) since the hold-up of microorganisms in the filter is large, the film thickness is large in the sense defined by Figure 6 .

When the filter is started up an accumulation of microorganisms takes place until the performance is essentially independent of additional accumulation. However, even when the characteristics of the inflow are constant it is found that the performance of the fermenter varies with time ${ }^{28,29}$ as new growth interacts with the liquid-flow pattern. Thus it has to be accepted that the performance of a 'trickling filter' varies with time about a mean. This variation is unpredictable and the objective must be to provide an approximation to the mean performance.

The liquid-flow pattern is inherently variable and in many instances is probably more characteristic of cascade flow than truly gravitational film flow $^{30}$. Because of the magnitude of the flow-rates used it is quite likely (a) that the performance is dominated by the microbial films, and (b) a liquidphase diffusional limitation exists. Because of the complexities of the flow and growth the liquid-phase mass-transfer coefficient verges on being incapable of interpretation and determination. However because of the 


\section{B. ATKINSON}

large microbial-film thickness, an asymptotic form of the biological rate equation finds application, i.e.

$$
N=\frac{k_{1}}{k_{2}} C^{*}
$$

It follows that

$$
N=h\left(C-C^{*}\right)=K_{(1)} C
$$

where $K_{(1)}$ is a first order rate coefficient

and

$$
\frac{1}{K_{(1)}}=\frac{1}{h}+\frac{1}{k_{1} / k_{2}}
$$

Insertion of equation 36 into 21 followed by integration leads to

$$
C / C_{\mathrm{I}}=1-\exp \left(-\frac{K_{(1)} A_{\mathrm{w}} Z}{Q}\right)
$$

where $A_{\mathrm{w}}$ is the biologically-active area per unit volume $\left(A_{\mathrm{w}}<A_{\mathrm{s}}\right)$.

Because the liquid-flow pattern is strongly influenced by the presence of the microorganisms, it is only possible to obtain realistic values of the masstransfer coefficient $h$ (contained in $K_{(1)}$ ), in the presence of microorganisms. The logical procedure to follow is to determine experimentally the 'overall volumetric rate coefficient' $K_{(1)} A_{\mathrm{w}}$ in a 'pilot' apparatus of the process geometry and to use these data for design purposes in conjunction with equation 38. This removes the necessity for separate determinations of $h$, $A_{\mathrm{w}}$ and the biological rate coefficients $k_{1}$ and $k_{2}$. It is of course to be expected that $K_{(1)} A_{\mathrm{w}}$ will vary in a complex manner with the flow rate due to changes both in the mass transfer coefficient $h$ and the interfacial area $A_{\mathrm{w}}$.

'Controlled' microbial film thickness.

The liquid flow-rates in a trickle-flow fermenter containing microbial films of controlled thickness are usually observed in laminar flow. Furthermore the microbial films have little or no effect on the liquid-flow patterns. In these circumstances the liquid-phase diffusion coefficient $h$ can be predicted from first principles using the conservation equation, the known hydrodynamics of liquid films and a knowledge of molecular diffusion.

Such predictions have been successfully carried out by Atkinson and coworkers $^{11.22,23}$ using both thin and thick microbial films in a biologicalfilm reactor and by Atkinson and Williams ${ }^{15}$ in a laboratory model of a trickling filter.

\section{DISCUSSION}

According to the proposed hypothesis regarding microbial adhesion, microbial hold-up on the solid surfaces of a fermenter plays a contributory part towards the performance of the fermenter. Since this hold-up is proportional to the product of the biological-film thickness and the surface 


\section{MICROBIAL FILMS IN FERMENTATION}

area, it follows that, on occasion, it can become a dominant feature. This appears to be the case in microbial leaching ${ }^{8}$ where the area term is large, and for those organisms which produce a large biological-film thickness. In studies using such organisms, mechanical devices akin to windscreen wipers have been added to fermenters in an attempt to control the films produced $^{16.17}$.

The theories presented represent logical extensions of the work by Herbert ${ }^{25.27}$ on microbial floc systems, and of that by Atkinson et al. ${ }^{1.19 .22}$ on microbial film systems. The question arises as to whether the biological rate coefficients for a given substrate-microbe system are the same for both flocs and films. While this information is unavailable at present, it seems reasonable to anticipate that any differences will be of relatively small proportions.

On the basis of the hypothesis regarding the adhesion of microorganisms to surfaces, the inclusion of biologically-active surface in a fermenter would appear to provide some considerable operational advantages providing the microbial-film thickness can be controlled. The completely-mixed microbial film fermenter represents one way in which a controlled microbial film can be added to a fermenter. The performance characteristics of this fermenter are in general agreement with the proposed theory, although some of the detailed assumptions made regarding mass-transfer coefficients, floc size etc. are only approximately true.

The operational characteristics of the CMMFF indicate the importance of the mechanical action of the support particles in the control of the biological film; this is in contrast to the contribution of fluid mechanical shear. This conclusion is emphasized by the extent of microbial growth in the transfer lines as compared with the fluidized-bed region of the fermenter.

The fluidized-bed fermenter, particularly when arranged in series with a microbial floc fermenter, provides a potential alternative to the 'tower' fermenter in reflecting the environmental history of a fermentation which characterizes batch fermentation.

In the case of fermenters which exploit microbial films, one of the criteria in the selection of a species and strain of microorganism for a particular conversion would necessarily involve the microbial film-forming properties of the organism. This is similar to the use of flocculating organisms in tower fermentation ${ }^{18}$.

The equilibrium biological-film thickness is, to some degree, a dependent process variable, since it is largely determined by the mechanical forces present in the fermenter. As such it can only be determined by studies on the particular substrate-microbe system and fermenter configuration involved. A numerical estimate of its value can be obtained either from the overall fermenter performance and developments of the theories as described, by an adaptation of the optical method used by Kornegay and Andrews ${ }^{12}$; or by some form of recovery procedure followed by gravimetric assay.

\section{ACKNOWLEDGEMENTS}

The author would like to thank V. T. Chue, C. S. Garland, S. L. Marshall, 


\section{B. ATKINSON}

C. N. Peridakis and M. J. Stride for their assistance with the experimentation reported in this paper.

\section{REFERENCES}

1 C. E. Zobell. J. Bacteriol, 46, 39 (1943).

2 D. H. Larsen and R. L. Dimmick, J. Bacteriol. 88. 1380 (1964)

${ }^{3}$ R. J. Munson. Chapter 2 Methods in Microbiolog! Vol. II (Norris, J. R. and Ribbons, D. W. Eds.). Academic Press. New York (1970).

${ }^{4}$ P. N. J. Chipperfield. in Water Pollution Control Engineering. (Downing. A. L.. Ed.), HMSO. London (1970).

5 J. A. Borchardt. Biotechnol. Bioeng. Symp. Series No. 2. 131, (1970).

6. C. Llaguno. Process Biochem. 6. 27 (1971).

- A. Rhodes and D. L. Fletcher. Principles of Industrial Microbiology. Pergamon Press, Oxford (1966).

${ }^{8}$ P. A. Trudinger, Minerals Sci. Eng. 3, 13 (1971).

${ }^{9}$ B. Atkinson. Chapter 5 : Biochemical Reaction Engineering in Chemical Engineering, Vol. III, (Coulson, J. M. and Richardson, J. F., Eds.). Pergamon Press, London (1971).

${ }^{10}$ N. Blakebrough, Chem. Engnr. (London). No. 258. CE 58 (1972).

1 B. Atkinson and I. S. Daoud. Trans, Inst. Chem. Engrs. 48, 245 (1970).

12 B. H. Kornegay and J. F. Andrews, J. Water Pollution Control Fed. 40. 460 (1968).

${ }^{13}$ H. R. Bungay. W. J. Whalen and W. M. Saunders, Biotechnol. Bioeng. 11, 765 (1969).

${ }^{14}$ H. A. Hawkes. The Ecology of Waste Water Treatment. Pergamon Press, London (1963).

15 B. Atkinson and D. A. Williams. Trans. Inst. Chem. Eng. 49, 215 (1971).

if P. A. Anderson, J. Gen. Physiol. 36, 733 (1953)

1 J. H. Northrop. J. Gen. Physiol. 38. 105 (1954).

${ }^{18}$ R. N. Greenshields and E. L. Smith. Chem. Engnr. (Londom). No. 249. CE 182 (1971).

${ }^{19}$ B. Atkinson and I. S. Daoud, Trans. Inst. Chem. Engrs. 46. 19 (1968).

${ }^{20}$ B. Atkinson. S. Y. How and I. J. Davies (to be published).

21 B. Atkinson, Biochemical Reactors, Pion Press, London (to be published 1973).

22 B. Atkinson, I. S. Daoud and D. A. Williams, Trans. Inst. Chem. Engrs., 46. 245 (1968).

${ }^{23}$ S. Y. How, Ph.D. Thesis, University of Wales, 1972.

${ }^{24} \mathrm{~B}$. Atkinson and S. Y. How. (to be published).

${ }^{25}$ D. Herbert, Soc. Chem. Ind. (London), Monograph No. 12. 21 (1956).

${ }^{26}$ B. Atkinson and I. J. Davies, Trans. Inst. Chem. Engrs. 50, 208 (1972).

27 D. Herbert, Progress in Microbiology. Symp. VI, p. 381, VIIth Int. Congr. Microhiology, Stockhoim (1958).

${ }^{28}$ G. E. Eden. K. Brendish and B. R. Harvey. J. Proc. Inst. Sewage Purification. 513 (1964).

${ }^{29}$ A. M. Bruce and J. C. Merkens. J. Inst. Water Pollution Control. No. 2, 69. 103 (1970).

${ }^{30)}$ B. Atkinson. A. W. Busch, E. L. Swilley and D. A. Williams. Trans. Inst. (he'm. Engrs., 45, 257 (1967). 